\title{
DIRECTORES FANTÁSTICOS Y DÓNDE ENCONTRARLOS: LECCIONES SOBRE LA PROMOCIÓN INTERNA DE DIRECTIVOS ELEGIDOS POR ALTA DIRECCIÓN PÚBLICA EN CHILE
}

\section{Sergio Galdames ${ }^{1}$ y Álvaro González ${ }^{2}$}

\begin{abstract}
RESUMEN
La relevancia del liderazgo para el mejoramiento del aprendizaje de los estudiantes es innegable, pero los estudios advierten de una crisis global en el reclutamiento y retención de directores debido a las complejidades asociadas al cargo. En Chile, la selección directiva favorece el reclutamiento de candidatos externos por sobre la promoción interna, lo que conlleva dificultades derivadas de la inserción en una cultura escolar nueva, en un contexto de alta rotación directiva. Este artículo presenta una mirada alternativa y, a través de un estudio de casos cualitativo, analiza la trayectoria de 10 directores de establecimientos municipales elegidos por Alta Dirección Pública (ADP), luego de un proceso de promoción interna. Los resultados se organizan utilizando el marco propuesto por Moorosi (2010): Anticipación, los futuros directores son identificados tempranamente, ejercen liderazgo de manera informal y acceden a oportunidades de aprendizaje profesional; Adquisición, los futuros directores acceden a puestos de liderazgo formal, desarrollando conocimientos profesionales y aumentando su visibilidad frente a la comunidad escolar; y Desempeño, los directores enfrentan la transición al cargo y deben manejar las relaciones con otros actores relevantes. A partir de estos hallazgos, se discuten cinco lecciones acerca de la promoción interna de líderes escolares de modo que complemente el reclutamiento externo en una futura carrera directiva en Chile.
\end{abstract}

Conceptos clave: carrera directiva, Chile, desarrollo profesional, liderazgo educativo, sucesión planificada.

\section{FANTASTIC PRINCIPALS AND WHERE TO FIND THEM: LESSONS ABOUT INTERNAL PROMOTION OF SCHOOL LEADERS SELECTED VIA THE SYSTEM FOR PUBLIC MANAGEMENT POSITIONS IN CHILE}

\section{ABSTRACT}

The importance of leadership for student learning is undeniable, but studies warn of a global crisis in the recruitment and retention of principals due to the complexities associated with this position. In Chile, principal selection favors the recruitment of external candidates over internal

1 Institute of Education-UCL, Londres, Reino Unido. Contacto: sergio.galdames.15@ucl.ac.uk

2 PontificiaUniversidad Católica de Valparaíso, Valparaíso,Chile. Contacto: alvaro.gonzalez@pucv.cl 
132 DIRECTORES FANTÁSTICOS Y DÓNDE ENCONTRARLOS: LECCIONES SOBRE LA PROMOCIÓN INTERNA DE DIRECTIVOS ELEGIDOS POR ALTA DIRECCIÓN PÚBLICA EN CHILE - S. Galdames y Á. González

promotion, which entails difficulties derived from the insertion into a new school culture. This article introduces an alternative perspective and, through a qualitative case study, analyzes the trajectory of 10 municipal school principals selected through Senior Public Management (ADP) after a process of internal promotion. Findings are organized using the framework proposed by Moorosi (2010): Anticipation, future principals are identified early, exercise leadership informally and access professional learning opportunities; Acquisition, future principals access formal leadership positions, developing professional knowledge and increasing their visibility with the school community; and Performance, principals face the transition to office and must manage relationships with other relevant actors. Based on these findings, five lessons are discussed for a strategy of internal promotion of future school leaders that can complement external recruitment for a future principal career in Chile.

Key concepts: Chile, education leadership, planned succession, principal career, professional development.

\section{Introducción}

La película Animales fantásticos y dónde encontrarlos (Heyman, Kloves, Rowling \& Wigram, 2016), acompaña la historia de Newt Scamander, un hechicero/zoólogo que viaja desde Londres a Nueva York con la misión de proteger animales mágicos. La historia que observamos en la película no es la de una persona cazando criaturas mágicas, sino identificándolas y protegiéndolas en un maletín para más adelante devolverlas a su hábitat. El maletín funciona como una especie de hospital móvil, que permite transportar a los animales mientras se recuperan física y emocionalmente. Quizás un nombre más preciso para la película podría ser: Animales fantásticos: rescátalos, cuídalos y devuélvelos.

La historia que la película narra nos puede ayudar a reflexionar en torno a la carrera de los directivos escolares desde una perspectiva diferente. En vez de poner la mirada en el contexto externo para hallar a un buen líder escolar, podríamos reconocer que los futuros líderes ya están en nuestras escuelas y liceos, y que es necesario identificarlos tempranamente, ayudarlos a desarrollarse en un ambiente protegido y, finalmente, ubicarlos en lo que será su hábitat: la dirección escolar. Este tema es de suma importancia, puesto que la evidencia en investigación es concluyente al señalar la relevancia de un buen liderazgo para el mejoramiento escolar y el aprendizaje 
de los estudiantes, siendo muy difícil pensar en el fortalecimiento de los sistemas escolares sin tener excelentes líderes en las escuelas (Leithwood, Harris \& Hopkins, 2008).

No obstante, son cada vez más los estudios que advierten acerca de una crisis global en el reclutamiento y retención de directivos escolares (Torres, 2018; White \& Cooper, 2011). La evidencia internacional muestra un creciente desinterés en postular a la dirección escolar, y, al mismo tiempo, se evidencia una mayor rotación y renuncia de directores y directoras (James et al., 2018). Los estudios sugieren una interdependencia entre los factores que moderan el interés por postular a la dirección con los que explican la rotación y renuncia, donde las difíciles y desafiantes condiciones laborales aparecen como las principales causas de este problema (Connolly, Milton, Davies \& Barrance, 2018; Earley \& Weindling, 2007; Stevenson, 2006).

Las implicancias de esta crisis directiva demandan con urgencia a los sistemas educativos la necesidad de innovar en sus estrategias para contar con suficientes directivos en todas sus escuelas, sin embargo, frecuentemente estas estrategias se han focalizado desproporcionadamente en el reclutamiento externo, dejando de lado la identificación de líderes al interior de las organizaciones escolares. En Chile, las leyes que regulan la selección directiva —Ley No 20.501 de Calidad y Equidad de la Educación y Ley No 20.903 de Carrera Docente-, no estimulan el desarrollo interno de líderes, promueven más bien el reclutamiento y selección de candidatos externos que cumplan con una serie de requisitos de formación, desempeño y experiencia profesional previa (Rivero, Hurtado y Morandé, 2018). Asimismo, en ausencia de una carrera directiva formal, las iniciativas actuales han abordado el desarrollo de futuros líderes a través de programas de formación donde cada postulante decide individualmente si cursarlos o no, sin proporcionar mecanismos efectivos que faciliten la postulación y acompañen la inserción en cargos directivos una vez finalizada su formación (Campos, Balborán, Bustos y González, 2014; Galdames, 2019).

Ante este escenario, muchos nuevos líderes chilenos se incorporan a establecimientos educacionales que les son desconocidos 
134 DIRECTORES FANTÁSTICOS Y DÓNDE ENCONTRARLOS: LECCIONES SOBRE LA PROMOCIÓN INTERNA DE DIRECTIVOS ELEGIDOS POR ALTA DIRECCIÓN PÚBLICA EN CHILE - S. Galdames y Á. González

y donde dedican los primeros años de ejercicio de la función directiva a sortear dificultades derivadas de su inserción en la cultura de la organización escolar, mientras navegan en las complejidades relativas al cargo de director o directora (Montecinos et al., 2015). Por ello, resulta relevante complementar los esfuerzos que ya se realizan en Chile para el reclutamiento externo de directores con la identificación temprana y promoción interna de futuros líderes escolares que ya se encuentran en los establecimientos, a fin de combatir el desinterés, rotación o renuncia de directivos escolares en nuestro sistema educativo.

Este artículo asume que, frente a la creciente escasez global de líderes escolares y las condiciones de política y normativa que favorecen el reclutamiento externo de directores, identificar y desarrollar a un futuro director al interior del establecimiento es un hecho fantástico. Es por ello que resulta relevante investigar la trayectoria de profesionales que se desarrollan como líderes al interior del mismo establecimiento que posteriormente dirigirán. De esta forma, este artículo se propone describir y analizar la trayectoria de directores de escuelas municipales escogidos por ADP, luego de un proceso de promoción interna, a través de un estudio cualitativo de 10 casos. Para analizar estas trayectorias se recurrió al marco analítico desarrollado por Moorosi (2010), describiendo el proceso de estos 10 directores en tres etapas: anticipación, adquisición y desempeño. A partir de los hallazgos, se discuten las implicancias que tienen para una posible estrategia de desarrollo de futuros líderes escolares al interior de los establecimientos y se proponen cinco lecciones que pueden complementar el reclutamiento externo de directivos en el contexto de una futura carrera directiva en Chile.

\section{Revisión de literatura}

\subsection{Crisis global en liderazgo educativo y sus efectos}

El problema que enfrentan los sistemas escolares para atraer y retener a sus directores no es nuevo. A comienzos de siglo, Hartle y Thomas (2003), a partir de evidencia demográfica, identificaron un gran problema en el futuro del liderazgo educativo en el Reino 
Unido. De acuerdo con sus estimaciones, la mayoría de los directores, subdirectores y líderes medios (por ejemplo, jefes de departamento o ciclo) estaban cercanos a la edad de jubilación y, adicionalmente, cada vez disminuían más los jóvenes interesados en ser profesores. Este desbalance de capital humano para proveer de líderes a las escuelas fue denominado por los autores como una "bomba de tiempo demográfica" (2003, p. 5), proponiendo el desarrollo de políticas públicas que impulsaran mecanismos de incentivo, como la mejora de las condiciones laborales y materiales para los directores. Las predicciones de este estudio fueron acertadas, ya que en la actualidad el Reino Unido se encuentra en una gigantesca crisis de profesionales tanto a nivel directivo como docente (Department for Education UK, 2019; James et al., 2018).

Algo muy similar sucedía cruzando el Atlántico. En la provincia de Ontario en Canadá, Fink y Brayman (2004) anticipaban un potencial problema en la provisión de directores escolares para sus escuelas al reconocer un creciente desinterés de subdirectores y líderes medios en ascender en la carrera directiva. En 2004, Ontario se preparaba para el éxodo de cerca del $70 \%$ de sus directores en los siguientes 10 años y, pero a diferencia del Reino Unido, esta provincia optó por la rotación o cambio planificado de directores luego de un periodo de tiempo, argumentando que "los directores bajan su eficacia luego de seis o siete años" (Fink \& Brayman, 2003, p. 442). Sin embargo, este supuesto fue cuestionado por la evidencia, al reconocer que la rotación sistemática no solo no tiene un impacto significativo en los aprendizajes de los estudiantes (especialmente en escuelas con dificultades), sino que también actúa como una barrera para la preparación de futuros líderes (Hargreaves, 2005).

Estos dos ejemplos ilustran la complejidad de abordar la crisis de liderazgo y ofrecen pistas acerca de las principales consecuencias de generar cambios sucesivos en el cargo de director. Por una parte, está bien documentado que las escuelas con una alta rotación directiva presentan mayor frecuencia de problemas en la cultura escolar e influencia negativa en los aprendizajes de los estudiantes (Meyer, MacMillan \& Northfield, 2009). Miller (2013) encontró que los aprendizajes de los estudiantes descendían significativamente 
136 DIRECTORES FANTÁSTICOS Y DÓNDE ENCONTRARLOS: LECCIONES SOBRE LA PROMOCIÓN INTERNA DE DIRECTIVOS ELEGIDOS POR ALTA DIRECCIÓN PÚBLICA EN CHILE - S. Galdames y Á. González

durante los primeros dos años después de la llegada de un nuevo director, y no retomaban la línea base hasta cinco años luego del cambio directivo. Hargreaves, Moore, Fink, Brayman y White (2003) sugieren que los directores tienen que estar un mínimo de cinco años para observar algún tipo de cambio significativo en la escuela, y que la salida anticipada de un director no solo puede detener el mejoramiento, sino que también retrocederlo.

Por otra parte, los procesos de reclutamiento, selección e incorporación directiva son extremadamente costosos para las autoridades educativas. En un estudio que analizó seis distritos en Carolina del Sur, Estados Unidos, Tran, McCormick y Nguyen (2018) estiman que el costo de reemplazar a un director es de USD $\$ 24.000$ aproximadamente, llegando en algunos casos a superar los USD\$50.000. Este cálculo no solo incluye el dinero usado directamente en el procedimiento, sino también los recursos complementarios como el tiempo de trabajo de profesionales de apoyo, reuniones de planificación e informes adicionales, los cuales muchas veces son invisibles en los cálculos finales.

\subsection{La crisis de liderazgo en Chile}

En Chile, Valenzuela, Allende y Vanni (2018) reportan que entre 2005 y 2015 el 60\% de los directores de escuelas y liceos públicos abandonó su cargo antes del quinto año de ejercicio de su función, y que casi la mitad de ellos no continuó trabajando en el sistema escolar. Similarmente, Donoso, Fernández y Reyes (2019) realizaron un estudio de caso cualitativo con 12 directivos que fueron seleccionados a través de un proceso de postulación y concurso público, y dejaron sus cargos antes de completar su primer contrato de cinco años. El estudio evidencia una serie de tensiones en el ejercicio profesional de estos directivos, principalmente a partir de políticas nacionales y locales que dificultaron el ejercicio de su función y, eventualmente, los llevaron a la interrupción de su carrera.

Anteriormente, un estudio longitudinal cualitativo que acompañó a 13 directores noveles durante sus primeros tres años en la dirección, explorando sus procesos de aprendizaje en el cargo, identificó dificultades en la retención de aquellos que asumían por 
primera vez dicho cargo. Uno de los resultados más llamativos del estudio en este aspecto apunta a que al final del segundo año, uno de los directores había renunciado, mientras que dos habían sido despedidos por las autoridades educativas locales (Galdames et al., 2018).

Los resultados de estos estudios cualitativos refuerzan los hallazgos de Valenzuela y su equipo (2018), y entregan mayor profundidad respecto de las complejidades del ejercicio profesional de los directores para explicar, al menos en parte, la alta rotación en nuestro país. Asimismo, esta evidencia sugiere potenciales problemas para los directores noveles, en especial para los que comienzan su rol directivo en una comunidad educativa que les es desconocida, lo que podría tener un impacto en el interés de futuros líderes por asumir la función directiva.

Al respecto, en un estudio publicado recientemente (Galdames \& González, 2016), se exploró el interés en postular al cargo de director de una muestra de 220 docentes chilenos. Si bien a nivel agregado se observó un interés relativamente alto en postular, los análisis mostraron que este es altamente moderado por la edad de los participantes y las oportunidades de ejercer liderazgo informal en la escuela. De esta forma, los docentes jóvenes que mantenían algún tipo de actividad como líder medio, formal o informal (jefatura de Unidad Técnica, departamento, ciclo, o coordinador de programas e iniciativas), manifestaron un alto interés en ser directores en el futuro; por el contrario, a medida que los profesores envejecían y concentraban sus responsabilidades en la enseñanza, este interés disminuía significativamente.

\subsection{La carrera directiva como carrera sin-bordes}

La investigación en torno al trabajo y la carrera profesional sugiere que la preocupación por la retención no es singular de los sistemas educativos, sino que es un tema compartido por la mayoría de las industrias (Guest \& Rodrigues, 2014; Kamp, Lambrecht \& Søndergaard, 2011). Las denominadas carreras sinbordes (boundaryless careers), reconocen un creciente abandono de la trayectoria laboral tradicional, caracterizada por el trabajo a perpetuidad en solo una organización y en la que un trabajador 
138 DIRECTORES FANTÁSTICOS Y DÓNDE ENCONTRARLOS: LECCIONES SOBRE LA PROMOCIÓN INTERNA DE DIRECTIVOS ELEGIDOS POR ALTA DIRECCIÓN PÚBLICA EN CHILE - S. Galdames y Á. González

gradualmente avanza en la escalera laboral aumentando su remuneración y beneficios, por una carrera flexible y móvil, que favorece la búsqueda de propósitos morales, aprendizaje personal y balance vida-trabajo (Arthur \& Rousseau, 1996; Briscoe \& Hall, 2006). En una carrera sin-bordes, la idea del cambio de trabajo, de rol, e incluso de disciplina es común, especialmente para aquellos individuos que se reconocen como altamente competentes y que han acumulado éxitos laborales (Verbruggen, 2012). Adicionalmente, las trayectorias laborales de carreras sin-borde han sido identificadas con mayor frecuencia en profesionales jóvenes, usualmente menores de 50 años (Seipert \& Baghurst, 2014; Torres, 2018).

Si bien los estudios referidos a la carrera sin-bordes no han sido aplicados directamente en el contexto educativo, sus hallazgos pueden ofrecer una perspectiva útil para analizar la carrera directiva. Primero, respecto del reclutamiento de futuros líderes, la evidencia señala que el impacto de aumentar las recompensas materiales para mejorar el atractivo de cargos directivos es limitado, siendo más importante alinear las responsabilidades laborales con los valores personales, oportunidades de aprendizajes significativos y con el logro de un equilibrio entre la vida personal y familiar si se quiere aumentar la retención de directores jóvenes (Edge, Galdames \& Horton, 2017). Segundo, en cuanto a la retención y recambio de directivos, la evidencia sobre carrera sin-bordes demandaría una reestructuración estratégica de las trayectorias laborales de los directivos, dado que los profesionales buscan constantemente nuevos desafíos que se traducen en periodos breves de trabajo en un mismo lugar (Suutari, Tornikoski \& Mäkelä, 2012). Por ello, es importante pensar en estrategias que incentiven el desarrollo de futuros líderes al interior de las organizaciones escolares, con el objetivo de planificar anticipadamente el recambio de directivos y reducir los periodos de ausencia de dirección formal, sin que ello implique una variación significativa de la calidad del liderazgo.

Respecto de este último punto, recientemente la Fundación Wallace publicó la evaluación de una iniciativa desarrollada entre 2011 y 2016 en seis distritos de Estados Unidos, llamada Principal pipelines o "chimenea de directores", que considera un conjunto de 
procedimientos y políticas para desarrollar y apoyar a líderes escolares efectivos. Uno de los pilares de la iniciativa es la identificación temprana y el acompañamiento al interior del centro escolar de futuros líderes, como una estrategia económicamente más eficiente que la sucesiva rotación de directores contratados externamente. La evaluación señala que las escuelas que crearon chimeneas de directores en grandes distritos superaron a otras similares en los distritos de comparación en resultados de lectura y matemáticas de sus estudiantes (Gates, Baird, Master \& Chavez-Herrerias, 2019). Esto abre la puerta para discutir acerca de la necesidad de diversificar las fuentes de reclutamiento de futuros directores.

\subsection{Promoción interna y reclutamiento externo de líderes}

Existe una larga discusión en torno a los beneficios y desafíos de elegir un líder desde la organización o importarlo externamente, tanto así que la evidencia no ha permitido encontrar una conclusión definitiva (Cannella \& Lubatkin, 1993; Legore \& Parker, 1997). Una de las preocupaciones centrales se relaciona con la pertinencia, es decir, cuándo es mejor la promoción interna o el reclutamiento externo. Por ejemplo, algunos estudios concluyen que las organizaciones con bajo desempeño tienden a preferir la contratación de líderes externos, buscando no solo un cambio en el funcionamiento organizacional, sino también implementar importantes y urgentes transformaciones culturales (Karaevli, 2007; Schwartz \& Menon, 1985). Sin embargo, los hallazgos de Santora, Caro y Sarros (2007) en organizaciones sin fines de lucro relativizan la decisión acerca de las ventajas del líder interno o del externo, dependiendo de las metas de la organización. La elección se sostiene, entonces, en el análisis de las necesidades organizacionales y el cuestionamiento de la existencia o no de un potencial líder interno que pueda alcanzarlas. Además, Agrawal, Knoeber y Tsoulouhas (2006) señalan que los candidatos externos son usualmente percibidos con mayores capacidades técnicas que los internos, lo cual explica el esfuerzo extra por realizar una búsqueda fuera de la organización. Sin embargo, los autores advierten acerca de posibles sesgos en el supuesto de que el éxito previo del candidato en otras organizaciones sea un predictor del desempeño futuro en la nueva organización. 
140 DIRECTORES FANTÁSTICOS Y DÓNDE ENCONTRARLOS: LECCIONES SOBRE LA PROMOCIÓN INTERNA DE DIRECTIVOS ELEGIDOS POR ALTA DIRECCIÓN PÚBLICA EN CHILE - S. Galdames y Á. González

Por otra parte, estudios enmarcados en una postura de cultiva tus propios líderes (grow your own leaders), que rescata ideas de la agricultura doméstica, destacan las ventajas de desarrollar internamente líderes considerando la cultura, necesidades y capacidades particulares de cada escuela, como también las ventajas económicas que ello conlleva (Tirozzi, 2001). Este enfoque también reconoce la importancia de establecer una relación honesta y bien planificada con los futuros candidatos y la comunidad educativa para facilitar la eventual transición y aumentar el compromiso organizacional de los futuros líderes (Joseph, 2009).

Esta información sugiere que la sucesión directiva debe ser considerada como un proceso clave del plan estratégico de cualquier organización escolar, fomentando la identificación y preparación adecuada de futuros líderes. Sin embargo, la evidencia reciente en Chile indica que más de un tercio de los directores en ejercicio no tuvo ninguna experiencia en liderazgo antes de ejercer por primera vez el cargo, y que un 78\% no contaba con ningún tipo de formación especializada en el tema (Rivero et al., 2018). Por ello, es de vital importancia conocer en profundidad las trayectorias o rutas de carrera que llevaron a los actuales directores a ocupar dicho cargo a fin de establecer algunas etapas o momentos que permitan identificar oportunidades para acceder a este con una experiencia y preparación adecuadas. Uno de los pocos modelos disponibles para analizar las rutas de carrera de directivos fue desarrollado por Moorosi (2010) en el contexto sudafricano para caracterizar las experiencias de directoras que se abrían paso en un contexto marcadamente machista. Este modelo distingue tres etapas o momentos en la ruta de carrera hacia la dirección de estas profesionales, con un énfasis particular en identificar las barreras y obstáculos que se les presentaban en cada una de ellas.

La primera etapa, anticipación, es caracterizada por la autora como el momento de desarrollo de capacidades técnicas y habilidades necesarias para asumir un rol de gestión y liderazgo al interior de sus establecimientos por parte de las futuras líderes, señalando la importancia de contar con una adecuada formación en el área y experiencia en roles formales e informales de liderazgo medio. La segunda etapa, adquisición, es el momento en que 
se busca activamente y se accede a cargos de dirección, donde la dimensión política de los procesos de selección de directoras se vuelve relevante y se vinculan fuertemente con la dimensión personal que considera las motivaciones, aspiraciones y redes de apoyo de las candidatas. La tercera etapa, desempeño, corresponde al momento en que estas asumen las funciones de directora, e involucra no solo la implementación de acciones propias del cargo, sino también el abordaje de distintas situaciones sociales y culturales al interior de las organizaciones escolares que ponen barreras y desafíos al ejercicio de su rol como mujeres directoras.

Este modelo de ruta de carrera resulta relevante para analizar el caso chileno, pues incorpora dos elementos que la investigación reciente identifica como clave para el desarrollo de líderes escolares efectivos: la experiencia previa en cargos de gestión, y la preparación especializada en liderazgo (Galdames, 2019; Rivero et al., 2018).

\section{Metodología}

El estudio de casos que se presenta en este artículo se desprende de una investigación cualitativa más amplia que uno de los autores condujo en los últimos años en torno a las trayectorias profesionales de 28 directores y directoras de establecimientos municipales, seleccionados por ADP, en el marco de lo establecido por la Ley $\mathrm{N}^{\circ}$ 20.501. El propósito particular de este estudio de casos es describir y analizar la trayectoria de directores de escuelas municipales escogidos por ADP, luego de un proceso de promoción interna.

Para alcanzar dicho propósito, se realizó una muestra intencionada (Schulz, 2013) a partir de un análisis exploratorio de los datos, seleccionando a 10 de los 28 casos que accedieron al cargo de director a través de una trayectoria de promoción interna. Esto significa que las 10 personas estudiadas trabajaron como docentes y/o docentes directivos en la misma escuela o liceo previo a ser seleccionados por ADP para dirigir dichos establecimientos. Estos establecimientos se encuentran en ocho comunas en distintas regiones del país, y en más de la mitad de los casos $(n=6)$ los participantes han trabajado solo en un par de establecimientos en su vida laboral, 
142 DIRECTORES FANTÁSTICOS Y DÓNDE ENCONTRARLOS: LECCIONES SOBRE LA PROMOCIÓN INTERNA DE DIRECTIVOS ELEGIDOS POR ALTA DIRECCIÓN PÚBLICA EN CHILE - S. Galdames y Á. González

mientras que el resto solo lo ha hecho en el mismo centro escolar que dirigen. La Tabla 1 describe algunas de las características de los participantes.

Tabla 1

Características demográficas de participantes del estudio

\begin{tabular}{lcclccc}
\hline Seudónimo & Edad Género & $\begin{array}{c}\text { Formación inicial o } \\
\text { especialidad }\end{array}$ & $\begin{array}{c}\text { Experiencia } \\
\text { profesional } \\
\text { (años) }\end{array}$ & $\begin{array}{c}\text { Años de } \\
\text { experiencia } \\
\text { previos a la } \\
\text { dirección }\end{array}$ & $\begin{array}{c}\mathrm{N}^{\circ} \text { de } \\
\text { los que han } \\
\text { trabajado }\end{array}$ \\
\hline 1. Oliver & 34 & H & Educación básica & 10 & 7 & 1 \\
\hline 2. William & 33 & H & Educación básica & 10 & 8 & 2 \\
\hline 3. Selina & 35 & M & Educación especial & 10 & 8 & 1 \\
\hline 4. Barry & 37 & H & Educación Física & 14 & 10 & 1 \\
\hline 5. Tony & 45 & H & Historia & 20 & 15 & 2 \\
\hline 6. Jessica & 46 & M & Educación especial & 21 & 17 & 2 \\
\hline 7. Carol & 50 & M & Educación parvularia & 25 & 18 & 2 \\
\hline 8. Hannah & 58 & M & Educación parvularia & 36 & 26 & 3 \\
\hline 9. Carice & 62 & M & Educación básica & 42 & 20 & 2 \\
\hline 10. Michele & 65 & M & Francés & 45 & 30 & 2 \\
\hline
\end{tabular}

Fuente: Elaboración propia.

\subsection{Métodos de producción y análisis de datos}

La producción de datos se realizó a través de entrevistas biográficas individuales en profundidad (Denzin \& Lincoln, 2000) a los participantes entre septiembre de 2017 y enero de 2018. En cada una se exploraron detalles de la vida personal y profesional de los participantes, con un énfasis particular en analizar sus trayectorias laborales desde sus comienzos como docentes hasta alcanzar la dirección. Las entrevistas tuvieron típicamente una duración aproximada de dos horas y fueron realizadas en los establecimientos donde los participantes ejercen como directivos. Cada entrevista se desarrolló como una conversación en torno a temas centrales que fueron previamente revisados por los participantes y registrados en una pauta; sin embargo, se adoptó una postura indagatoria y flexible respecto del relato que cada participante ofrecía de su historia personal y trayectoria laboral.

El audio de las entrevistas fue grabado y luego transcrito, para someter dichas transcripciones a un análisis temático (Clarke 
$\&$ Braun, 2017), buscando patrones en las experiencias, discursos y prácticas en la carrera de los directores. Se organizó la información producida con los directivos de este estudio de acuerdo con las etapas del modelo de ruta de carrera de Moorosi (2010), y en consideración con los objetivos de este artículo, se expandió el sentido del modelo original para representar no solo las barreras que los directores estudiados experimentan en cada etapa, sino que también las oportunidades que les permitieron progresar hacia el cargo directivo que ocupan. Usando las tres etapas inicialmente desarrolladas por Moorosi (2010) fueron desarrollados nueve temas.

- Anticipación: identificación temprana, mentoría, liderazgo medio informal, y aprendizaje formal;

- Adquisición: liderazgo medio formal, visibilidad, y conocimiento profesional y de la cultura escolar;

- Desempeño: paso del bastón, y la comunidad se rompe.

\section{Resultados}

En esta sección se presenta información producida sobre los 10 casos analizados, organizada en función de las tres etapas del modelo de Moorosi (2010): anticipación, adquisición, y desempeño.

\subsection{Anticipación}

\subsubsection{Identificación temprana}

De acuerdo con la experiencia de los casos estudiados, la etapa de anticipación se asocia con un momento específico de identificación de estos profesores como potenciales líderes, inicialmente vinculado con un buen desempeño en el aula y con la demostración de responsabilidad profesional. En muchas ocasiones, este reconocimiento ocurrió durante el primer o segundo año en el puesto y provenía principalmente de los directivos, en muchas ocasiones del mismo director. De acuerdo con los participantes, la principal consecuencia de este reconocimiento fue motivacional, ya que se sintieron valorados, respetados y motivados a seguir desarrollándose. 
144 DIRECTORES FANTÁSTICOS Y DÓNDE ENCONTRARLOS: LECCIONES SOBRE LA PROMOCIÓN INTERNA DE DIRECTIVOS ELEGIDOS POR ALTA DIRECCIÓN PÚBLICA EN CHILE - S. Galdames y Á. González

La identificación temprana no fue solo palabras de aliento, sino que derivó en acciones y oportunidades de desarrollo posteriores.

ahí comencé reemplazando a la profesora que había salido jubilada y de ahí no me sacó nadie, empecé a hacer clases de todo porque me lo solicitó el director que estaba en ese momento, fue mi mentor, fue una gran persona porque él me guio, él me dijo 'William, ¿sabes qué?, estudia administración porque tú tienes pasta de líder' (William).

el director que estaba aquí, tenía esa capacidad de percepción de ver a la gente que era líder, entonces él me empezó a percibir del inicio y me hacía cargo de cosas, aunque yo viniera poquito, y después existió la posibilidad de que viniera con más horas a la escuela, y él en ese momento me hace cargo de la subvención escolar preferencial, me hace cargo de todo el PME [Plan de Mejoramiento Educativo] (Selina).

\subsubsection{Mentoría}

Como parte de la etapa de anticipación, y en paralelo con la identificación temprana, los casos estudiados indican que los futuros líderes conformaron duplas de trabajo con un directivo de su establecimiento. Algunos participantes indican que, por ejemplo, comenzaron a asistir al director en tareas formales, apoyando en la administración de la escuela o en la formulación de ideas y proyectos.

El desarrollo de estas duplas de trabajo es reconocido por los participantes como un espacio de desarrollo profesional continuo, con ganancias mutuas tanto para el directivo como para el futuro líder. Asimismo, asumir estas responsabilidades complementarias al liderazgo expandieron la identidad y también el rol del entonces docente, quien fue gradualmente abandonando la sala de clases como espacio de desempeño profesional, si bien este proceso en la mayoría de los casos demoró varios años.

[El director anterior] se fue y todo y yo creo que en cierta forma yo aprendí a querer esta parte de la administración por él, porque él me fue metiendo en esto, él me llamaba de a poco para que le 
ayudara a pasar asistencia, para que le ayudara con los proyectos, a redactar, después a pasarlos al computador, de a poco me fui metiendo a toda esta parte de la parte como administrativa del colegio, él también me fue delegando, después él me dio la coordinación de SEP [Subvención Escolar Preferencial] (Jessica).

[La jefa de Unidad Técnica Pedagógica] respetaba mucho también mis sugerencias, y fuimos tomando tantas cosas nosotros dos, en términos organizativos, que muy pocas cosas pasaban por el director: plan de mejoramiento, las compras SEP las veía yo todo lo que tiene que ver con lo técnico, lo pedagógico (Barry).

Como ilustran las citas anteriores, el proceso de mentoría comenzó, en la mayoría de los casos, bajo el rol de ayudantías en temas administrativos simples, gradualmente avanzando hacia temas más complejos con un mayor componente pedagógico. Para muchos de los participantes, este trabajo colaborativo los benefició en el sentido de ofrecer las oportunidades que necesitaban para ganar experiencia y confianza en sus capacidades como líderes bajo la tutela de un líder formalmente reconocido en sus establecimientos.

\subsubsection{Liderazgo medio informal}

En la mayoría de los casos estudiados, las distintas olas de reformas de las últimas dos décadas abrieron oportunidades para asumir roles informales de liderazgo al interior de cada escuela en esta etapa de anticipación. Por ejemplo, el Sistema de Aseguramiento de la Calidad y la Gestión Educativa (SACGE) a principios de siglo y, posteriormente, la Ley de Subvención Escolar Preferencial (SEP) (Ley $N^{\circ} 20.248$ ) generaron espacios de mayor participación para profesionales con experticias con las que los líderes formales no contaban en el momento. De este modo, la mayoría de los participantes pasó de apoyar a una dupla, equipo o un líder formal, a liderar iniciativas y proyectos con creciente autonomía, e incluso a representar a sus establecimientos fuera de ellos.

ahí asumí esa responsabilidad de llevar el plan de mejoramiento, la elaboración, después antiguamente, había que presentar toda la evidencia, ahora se está haciendo como más amigable el sistema 
146 DIRECTORES FANTÁSTICOS Y DÓNDE ENCONTRARLOS: LECCIONES SOBRE LA PROMOCIÓN INTERNA DE DIRECTIVOS ELEGIDOS POR ALTA DIRECCIÓN PÚBLICA EN CHILE - S. Galdames y Á. González

de la plataforma y siempre lo llevé, entonces armé un equipo bien bueno de profesores jóvenes (Carol).

me dice [la directora], te vas a hacer cargo de la matrícula, imagínate, así que de hacer clases, ser asesora del centro de alumnos, de repente de la noche a la mañana eres responsable del proceso de matrícula en el colegio (Michele).

Usualmente, los directores asignaron desafíos de mayor complejidad a los futuros líderes quienes, si bien sorprendidos inicialmente, respondieron tomando cada petición como una oportunidad de aprendizaje y desarrollo profesional. El paso de apoyar iniciativas a liderarlas no fue fácil para ningún futuro director, pero la asignación de una responsabilidad superior y novedosa siempre contó con el apoyo continuo de los directivos de los participantes de este estudio, tanto técnica como emocionalmente.

\subsubsection{Aprendizaje formal}

Finalmente, durante la etapa de anticipación, el reconocimiento de los futuros líderes por parte de los líderes formales también abrió opciones de aprendizaje profesional. Por una parte, los futuros líderes fueron animados a participar en programas de administración y liderazgo educativo; por otra, la relación con los directivos facilitó el acceso a financiamiento y a las condiciones laborales para desarrollar la formación.

él sabía para dónde yo iba, así es que él, bien perceptivo, bien visionario, me dice 'señorita me llegó una información [Programa Directores de Excelencia]', y me la pasa... 'yo quiero postular', 'postule', me dijo, 'yo la apoyo en todo', y postulé. Me favoreció mucho haber tenido toda esta experiencia, todo lo que es plan de mejoramiento, aparte que yo también tenía del otro lado, que nunca me quedaba muy tranquila. Me llama un día en la noche y me dice 'señorita, usted quedó de entre 15 mil y salió beneficiada y créalo' y yo creo que él estaba así, como un papá chocho (Selina).

En ese tiempo empezamos a estudiar licenciatura, el magíster después... empezamos a estudiar el magíster... ir a la escuela... 
lo hicimos en esta escuela, entonces igual empezaron cosas... igual me hicieron como resaltar un poco dentro del grupo... el grupo de los profesores y eso me favoreció, porque yo creo que eso me ayudó a que ellos me vieran como... como que yo era la más bacán, así como una cosa así, como que yo sabía más que el resto, entonces como que igual me preguntaban, me pedían la opinión (Jessica).

El incentivo a participar en estudios de posgrado fue una tendencia común en los participantes, pero el elemento clave fue que la formación sugerida a los futuros líderes estuvo altamente vinculada con las responsabilidades que comenzaban a asumir y los desafíos de su establecimiento. Por una parte, el aprendizaje formal fue una oportunidad de analizar y fortalecer su establecimiento, pues muchas veces requerían usar datos, desarrollar proyectos y trabajar con otras personas de la escuela. Por otra parte, directivos, docentes e incluso estudiantes y apoderados estaban al tanto de la formación que cursaban los futuros líderes, y los sacrificios que significa a nivel personal (por ejemplo, tener clases los sábados). La participación en estas instancias de aprendizaje profesional fue una declaración explícita y pública hacia la comunidad educativa de la decisión de los participantes de convertirse en futuros directivos.

\subsection{Adquisición}

\subsubsection{Liderazgo medio formal}

De acuerdo con los participantes del estudio, un momento clave para el avance en la sucesión interna fue el paso de trabajar junto con los directivos y asumir responsabilidades informales de liderazgo en sus escuelas, a ocupar un puesto formal de liderazgo medio como parte del equipo directivo en el mismo establecimiento. Este paso se fundamentaba, de forma similar a lo que sucedía con la identificación temprana en la etapa anterior, en una evaluación positiva del trabajo que realizaban estos futuros líderes, tanto en su desempeño en el aula como en la realización de tareas de gestión y liderazgo fuera de este espacio, e inclusive fuera de su establecimiento. 
Entré como profesor, fui tomando algunas responsabilidades, fui encargado del Centro de Padres, empecé a hacer funcionar cosas, entonces, eso, el director creyó en mí, y me puso de [jefe de] UTP. Y ahí, al momento de ser UTP, tuve que adentrarme en otro mundo que era el tema del currículum, el tema del liderazgo, él muchas veces delegaba temas en mí, entonces fui aprendiendo harto con él, y aparte este director, yo siento que ha sido como mi padre en el área profesional en cuanto a la pedagogía (Oliver).

yo le dije a la directora, sabes que encuentro que hago tanto, yo estoy media cansada porque el kínder, aunque yo tenía una muy buena asistente, en ese intertanto también me llaman a nivel regional a ser monitora del plan de fomento lector comunal y tenía que salir del colegio y hacer capacitaciones a mis mismos colegas de nivel, me tocaron comunas, tuve que trabajar en [comuna cercana] con mis colegas, yo le digo '[nombre directora] ¿sabes qué?, estoy como un poquito cansada, ¿por qué no ves la posibilidad que me dejen como UTP?' (Carol).

Estas primeras nominaciones formales de los futuros líderes se ven marcadas por un proceso de validación y acompañamiento del director o directora del centro escolar, con quienes ya se ha establecido una relación de confianza, cercanía y consejo. En cierto modo, la experiencia de los casos analizados muestra la importancia que hasta este punto tiene el vínculo con el director o directora para progresar en la ruta de carrera y la promoción interna de estos líderes.

\subsubsection{Visibilidad}

Según el modelo de Moorosi (2010), en la etapa de adquisición, quienes luego se convertirán en directores toman la decisión de postular al cargo sobre la base de los conocimientos y experiencia que han desarrollado en la etapa de anticipación. Sin embargo, en los casos aquí analizados, encontramos que también resulta clave la visibilidad que logran estos profesionales dentro de su comunidad educativa. La participación continua en actividades fuera de los límites de la sala de clases les otorgó a los futuros líderes la oportunidad de conocer a otros y de ser conocidos por otros. En espacios formales e informales, por años estos futuros líderes pudieron demostrar 
públicamente sus capacidades, compromiso e ideas a una comunidad educativa extendida, que gradualmente comenzó a validar su trabajo y su liderazgo, lo cual fue la piedra angular del interés en una futura postulación.

Apareció que la directora se tenía que jubilar un día y ella en una reunión dice que había un concurso público, una llamada a concurso y nosotros lo conversamos entre las colegas que funcionábamos ahí y todas empezaron 'hazlo tú', yo estaba haciendo un magíster en ese tiempo en [nombre ciudad], entonces 'tírate tú', que sí, que no, 'ya vamos, vamos a la pelea', dije (Hannah).

Se abre el concurso y yo le pregunto, y en eso siempre también fui leal a los jefes que he tenido, y le dije 'usted profesor [nombre director] ¿se va a postular?', porque yo también quería que él siguiera, que para mí trabajar con ellos nunca ha sido un pesar, siempre fue un alivio trabajar con ellos y le dije si quería postular y me dijo que no quería postular y me motivó a postular, y él años antes me fue formando, también él fue un hombre súper visionario y en eso me fue formando y también inculcando, 'cuando tú salgas tienes que hacer esto', de a poquito fue inculcando y claro cuando llegó el momento, me dijo que postulara, 'postula, postula, tú eres la persona que tiene que seguir acá', postulé y me fue bien dentro de todo [...] producto como te decía de lo que hizo este profesor, que fue mi director (Oliver).

Un elemento común que se aprecia en la experiencia de estos profesionales es que su decisión de postular al cargo de director estuvo fuertemente marcada por el paso al costado que dio el director o directora con quienes trabajaron previamente, facilitando su acceso al cargo. La figura de estos directores se posiciona como un respaldo formal a la voluntad de postular al cargo y una señal a otros miembros de la comunidad educativa que refuerza la visibilidad que ya habían alcanzado estos futuros líderes. 
150 DIRECTORES FANTÁSTICOS Y DÓNDE ENCONTRARLOS: LECCIONES SOBRE LA PROMOCIÓN INTERNA DE DIRECTIVOS ELEGIDOS POR ALTA DIRECCIÓN PÚBLICA EN CHILE - S. Galdames y Á. González

\subsubsection{Conocimiento profesional y de la cultura escolar}

Durante la etapa de adquisición, la postulación formal de los directores estudiados se vio favorecida por el alto conocimiento que estos tenían acerca de la cultura escolar local. Este conocimiento se expresaba como una clara noción de los desafíos y oportunidades de la escuela a la que aspiraban liderar. De forma complementaria, los futuros líderes ya contaban con un conocimiento profesional de lo que implicaba liderar día a día un centro escolar. Ambos tipos de conocimiento son resaltados como una ventaja comparativa frente a otros postulantes.

una de las cosas que para mí fue fortaleza, es que yo ya conocía el colegio y conocía las cosas buenas y las cosas malas, las fortalezas y las debilidades que tenía, por lo tanto, yo estaba clarito ya con toda esta revolución que pasó por mi cabeza, qué es lo que tenía que hacer, qué es lo que hay que hacer, cuál iba a ser... mi modo de liderazgo, (...) según el contexto, eso es súper importante. Entonces uno tiene la capacidad, la competencia para ver qué tipo de liderazgo vas a utilizar en cada una de las escuelas... y yo lo tenía así como súper claro y ahí gané ese verano (Barry).

yo llevaba más tiempo, me manejaba en algunas cosas del colegio y yo siempre digo, 'en el país de los ciegos, el tuerto es rey', entonces me tocó esa responsabilidad de asumir, que manejaba muchas cosas y ahí yo creo que también por ejemplo, un poco de intrusa, de haber ido a otros perfeccionamientos, de conocer más allá, igual a uno le faltan cosas, pero sí sé que la práctica también te ayuda mucho, el trabajo in situ te ayuda mucho en tu parte profesional, (...) el trabajo diario te da muchas herramientas para resolver problemas cotidianos (Carol).

La combinación de los dos tipos de conocimiento, el profesional de tipo técnico acerca del ejercicio del cargo, y el de la cultura escolar de tipo más bien político en función de las relaciones e intereses de los actores de la comunidad educativa, permitieron a estos profesionales presentarse como candidatos fuertes al cargo. Asimismo, este conocimiento combinado también facilitó que los postulantes pudieran proponer un proyecto de dirección claramente 
alineado con las necesidades de la escuela, la cultura escolar y sus propios valores personales.

\subsection{Desempeño}

\subsubsection{Paso del bastón}

Como se presentó en el punto anterior, las etapas de anticipación y adquisición fueron desarrolladas en conjunto entre el futuro y el antiguo director, con el apoyo de la comunidad educativa. Gracias a ello, la última etapa de desempeño se benefició de una transparente e informada transferencia del poder. Si bien existieron momentos iniciales de subrogancias, eventualmente todos los participantes fueron seleccionados por ADP. En muchas oportunidades, el cambio de dirección fue recibido con alegría por el director saliente, dando un apoyo simbólico que facilitó las primeras acciones del nuevo director. Además, en la gran mayoría de los casos, el director saliente pasó temporalmente a ser parte de la planta directiva, tomando un rol formal menor (usualmente de inspector), pero informalmente asumiendo una mentoría constante al nuevo líder.

E: ¿Qué paso con el exdirector?

O: Es mi [jefe de] UTP.

E: ¿Está acá? Pensé que esta historia terminaba con él jubilado.

O: Claro que él igual como que quiere como dejarnos, pero no es porque tengamos algún problema y como que él no quiere dar paso a los más jóvenes, entonces hemos armado un equipo dentro de la escuela, somos tres personas, está la encargada del programa de integración, entonces somos los tres que trabajamos básicamente en el liderazgo de la escuela (Oliver).

me llama y me dice 'iestas sentada?', 'en realidad' le dije, 'estoy tirada en mi cama', 'ah, qué bueno, porque sabes que yo ya no soy más la directora', 'no te puedo creer', 'sî' me dice, 'y isabes quién es?', 'no' le dije yo, 'tú', '¿yo?', y yo lloré y lloré de pena porque que no fuera a pensar que yo le había hecho algo, porque como hubo cambio de gobierno y era un gobierno que a mí... era de mi lado, 'no', me dijo 'si estoy feliz, si vamos a seguir, yo voy a seguir siendo tu jefa de UTP y vamos a cambiar los roles' (Carol). 
152 DIRECTORES FANTÁSTICOS Y DÓNDE ENCONTRARLOS: LECCIONES SOBRE LA PROMOCIÓN INTERNA DE DIRECTIVOS ELEGIDOS POR ALTA DIRECCIÓN PÚBLICA EN CHILE - S. Galdames y Á. González

Las experiencias de estos 10 directores muestran que las relaciones entre líderes salientes y entrantes —que pueden convertirse en uno de los principales problemas en sucesión interna-, se aminoran por la historia y cercanía personal y profesional entre ambos. Los casos también ilustran que cuando la sucesión interna es planificada y se ejecuta adecuadamente, estos problemas no solo se reducen, sino que aparecen oportunidades importantes para la continuidad de procesos de mejoramiento en los centros escolares.

\subsubsection{La comunidad se rompe}

Finalmente, uno de los últimos temas que encontramos en la ruta de carrera de estos directores no proviene del interior del centro escolar, sino desde fuera. Acostumbrados a lo largo de su carrera a procesos de colaboración, apoyo, desafíos, lecciones y aprendizajes con sus antiguos directivos, al ocupar ahora el cargo de director, sus nuevos jefes directos, los respectivos jefes de educación municipal aparecen como una fuente constante de obstáculos y dificultades. En nueve de los 10 casos estudiados, la falta de colaboración desde este nivel intermedio del sistema es identificado como el principal problema para los nuevos directores en ejercicio.

E: ¿Qué es lo peor de las condiciones laborales?

H: La relación con el jefe, porque yo creo que ellos también son como muy de escritorio los jefes, me refiero a lo que hacen los DAEM [Departamento de Administración Educacional Municipal]. Yo se los he dicho, se los he dicho en muchos tonos, falta la parte humana de ellos (Hannah).

si tú me preguntas de este gerente [de Corporación de Educación Municipal], ¡cero, cero!, yo en las primeras reuniones lo cité, hice que nos reuniéramos, que todos los directores se alinearan conmigo, los del ADP, 'ya chiquillos hay que pedir reunión, esta cuestión no está funcionando, no está bien, no están entregado los materiales', es que siempre está ocupado él [gerente], no ha hecho nada... 'es que tengo hoy día una reunión, así que tengo un ratito para ustedes'... no se han dado [las condiciones] (Carice). 
La particular frustración para estos directivos promovidos por sucesión interna es la costumbre que tenían de trabajar colaborativamente con un superior, buscando no solo recursos sino espacios de reflexión y aprendizaje. Los casos estudiados identifican obstáculos para la ejecución de acciones y proyectos, pero también el reconocimiento de una desalineación de visiones y capacidades con los líderes intermedios encargados de la administración municipal de educación. En estos casos, los directores de sucesión interna no solo buscan un administrador educacional, sino un socio técnico pedagógico para avanzar en la mejora de sus centros escolares.

\section{Discusión: consideraciones para la promoción o sucesión interna de directivos}

En este artículo hemos revisado la experiencia de un grupo de 10 directores de escuelas municipales escogidos por ADP, para estudiar un aspecto poco investigado acerca de la sucesión directiva en centros escolares chilenos: la promoción interna de directivos. Hemos llamado a estas personas, directores fantásticos, no por su desempeño, sino por el valor que tiene analizar sus trayectorias para comprender con mayor profundidad este fenómeno. La estrategia de promoción interna no se encuentra libre de dificultades y siempre está presente el sesgo de que los nuevos líderes mantendrán la cultura organizacional en las cuales han sido socializados, mientras que los líderes externos están más dispuestos a la innovación y el cambio (Carlson, 1961; Miskel \& Cosgrove, 1985). Los casos estudiados fueron analizados a partir del modelo de tres etapas propuesto por Moorosi (2010), identificando las características de este proceso en cada una de ellas. A partir de estos hallazgos, a continuación, se discuten algunas de las lecciones para una posible estrategia de desarrollo de futuros líderes escolares en Chile a través de promoción interna, que pueda complementar los mecanismos de reclutamiento externo ya existentes.

\subsection{El tabú de la sucesión interna}

Uno de los componentes que dificulta cualquier estrategia de sucesión, pero en especial la interna, es que sea confundida con 
154 DIRECTORES FANTÁSTICOS Y DÓNDE ENCONTRARLOS: LECCIONES SOBRE LA PROMOCIÓN INTERNA DE DIRECTIVOS ELEGIDOS POR ALTA DIRECCIÓN PÚBLICA EN CHILE - S. Galdames y Á. González

prácticas poco éticas, o al menos cuestionables, de elección de un sucesor bajo los sesgos personales del director saliente. Travis (2016) señala que las sucesiones internas son influenciadas por el "miedo al amiguismo y favoritismo y clonación que puede llevar a la exclusión de minorías" (p. 9). Esto requiere llevar la sucesión como una práctica transparente y conocida por la organización (Hargreaves et al., 2003), tal como se observa en las etapas de anticipación y adquisición en los casos analizados. A nivel escolar, esto requiere que la escuela tenga claro su norte, misión y visión, discutiendo cómo cada líder contribuye a alcanzar los objetivos propuestos.

\subsection{Legado del líder anterior}

Vinculado con el punto previo, el éxito de cualquier estrategia de sucesión estará moderado por las características de la trayectoria del líder saliente (Oplatka, 2012). Johnson y Licata (1995) encontraron que el éxito de un nuevo director se relaciona fuertemente con los éxitos y fracasos del anterior. Esto puede ser usado como ventaja o desventaja para sucesores internos o externos, pero es indudable que los sucesores internos tendrán un acceso más expedito a esta información y más espacio de preparación que sus pares externos (Miskel \& Cosgrove, 1985). Esto se observa en los casos estudiados principalmente en las etapas de adquisición y desempeño, donde los futuros líderes logran una comprensión profunda de las oportunidades y desafíos de su comunidad educativa, y se valen del respaldo y acompañamiento de los líderes salientes, quienes incluso continúan como colaboradores en los primeros años de ejercicio de algunos directivos.

\subsection{Conocimiento del y sobre el nuevo líder}

Las investigaciones directivas son claras en señalar que un nuevo líder puede necesitar años para conocer la organización antes de poder implementar cambios y mejoras. Como lo señala Earley y Weindling (2007) en su etapa de "tomar el control" (p. 75), los directores pueden pasar largos periodos tratando de decodificar la cultura escolar antes de poder tener la atribución de generar procesos de cambios significativos. Cuando la mayoría de los docentes conoce al nuevo líder, las interrupciones y resistencias en el trabajo son menores; por el 
contrario, cuando solo algunos conocen al nuevo líder, los problemas y las emergencias puede ser más recurrentes. Es indudable que la sucesión interna genera ventajas en esta dimensión, ya que no solo el nuevo director conoce en detalle las oportunidades y desafíos de los docentes, sino que también la comunidad educativa tiene un juicio ya establecido respecto del nuevo líder. En los casos estudiados esto se observa principalmente en las etapas de anticipación y adquisición, donde el contacto con miembros de la comunidad escolar en roles informales, y la visibilidad que logra posteriormente en roles de liderazgo medio formal, se vuelven elementos fundamentales en su paso a asumir la dirección.

\subsection{El desafío de la inducción}

Uno de los elementos más estudiados en la sucesión directiva son los procesos de inducción para los nuevos líderes, los cuales paradójicamente son identificados de manera universal como fundamentales, pero muy poco frecuentes en contextos organizacionales y escolares (Bush \& Oduro, 2006; Moorosi, 2012). En Chile, otros estudios han encontrado situaciones similares, ya que escasean los programas de inducción formal o informal para directores y directoras en sus nuevas escuelas (Galdames et al., 2018). Los 10 directores estudiados en este artículo, por el contrario, presentan la ventaja de que durante las tres etapas de su trayectoria de promoción interna fueron progresivamente aprendiendo el cargo de dirección, incluyendo la etapa final de desempeño. Por lo demás, a partir de esta inducción progresiva, los líderes internos pueden comenzar a instalar procesos de cambio y mejoramiento mucho más rápido, ya que conocen las rutinas organizacionales y la cultura de la comunidad (Zepeda, Lanoue, Price \& Jimenez, 2014).

\subsection{Compromiso organizacional}

La investigación sugiere que la sucesión interna promueve la fidelidad organizacional, es decir, evita que las personas abandonen la organización (Miskel \& Cosgrove, 1985). Asimismo, existe evidencia de que la sucesión interna fomenta el desarrollo organizacional, mientras que la sucesión externa promueve el desarrollo individual (Carlson, 1961). En el caso de las trayectorias de promoción interna 
156 DIRECTORES FANTÁSTICOS Y DÓNDE ENCONTRARLOS: LECCIONES SOBRE LA PROMOCIÓN INTERNA DE DIRECTIVOS ELEGIDOS POR ALTA DIRECCIÓN PÚBLICA EN CHILE - S. Galdames y Á. González

de líderes, esto significa que se reduce el riesgo de perder a un nuevo director frente a una oferta laboral con mejores condiciones. Como hemos mencionado anteriormente en este artículo, la alta rotación directiva es una amenaza al mejoramiento educativo de las organizaciones escolares. En los casos estudiados, se observa que los futuros líderes se desarrollan a través de las tres etapas con una clara meta en mente: dar continuidad y mejorar los proyectos educativos de sus establecimientos, generando un intenso compromiso con sus comunidades y comprometiéndose decididamente con ellas.

\section{Conclusiones, limitaciones y futuras investigaciones}

No existe un acuerdo definitivo acerca de cómo desarrollar directores efectivos que movilicen la mejora educativa de escuelas y liceos. La investigación ha destacado la contribución de estrategias formales de desarrollo profesional para líderes escolares, como programas de posgrado, trabajo en red, mentorías, coaching y pasantías, entre otras (Irvine \& Brundrett, 2017; Reeves, 2010; Tulowitzki, 2013; Zepeda et al., 2014). Si bien no hay claridad absoluta respecto de cuál estrategia es la mejor, sí hay consenso en que cualquiera de estas opciones requiere mucho tiempo de preparación; algunos autores sugieren que tomaría varios años e incluso décadas formar a un líder de excelencia (Bower, 2007; Bush, 2011). Por otra parte, la evidencia señala que el interés por ocupar cargos de dirección en las escuelas y liceos ha decaído en las últimas décadas, dadas las complejidades que ello involucra, más aún cuando se trata de insertarse en establecimientos de los cuales se tiene poco conocimiento y donde los directivos deben pasar por difíciles procesos de socialización en la cultura escolar local (Earley \& Weindling, 2007; Galdames \& González, 2016; Stevenson, 2006). Todo esto sustenta la relevancia de describir y analizar la trayectoria de los directores de escuelas municipales chilenas, escogidos por ADP luego de un proceso de promoción interna.

Es importante, sin embargo, reconocer algunas limitaciones del estudio aquí presentado. En primer lugar, la selección de participantes para este estudio exploratorio restringe la generalización de los hallazgos más allá de los contextos en los que estos se desempeñan como directores. Si bien es posible encontrar patrones comunes en los 
10 directores estudiados, es necesario probar su validez con directores que cuenten con trayectorias similares de promoción interna en otros contextos (comunales o de tipo de sostenedor), e incluso comparar con las trayectorias de directores que son seleccionados a través de reclutamiento externo a fin de contrastar.

Por otra parte, los métodos cualitativos utilizados para la producción y análisis de datos e información, si bien son apropiados para un estudio exploratorio y analítico como este, tampoco permiten establecer afirmaciones más allá del alcance de los casos estudiados. Más aún, existe la posibilidad de ciertos sesgos de parte de los participantes debido a que se recurre a la memoria de eventos personales que sucedieron varios años antes de la producción de información. Por ello, es relevante que futuros estudios amplíen la muestra de participantes y complementen los métodos cualitativos con otros cuantitativos que permitan probar o refutar algunas de las hipótesis que se han levantado en este estudio, e idealmente comparar entre distintos grupos de directores con diferentes trayectorias profesionales.

Finalmente, las cinco lecciones que se desprenden de los hallazgos en relación con los 10 casos analizados pueden ser de utilidad para sentar las bases y lineamientos que contribuyan a una futura carrera directiva en Chile que considere trayectorias de sucesión o promoción interna como complemento de las estrategias de reclutamiento externo que ya se encuentran consideradas en la legislación y política educativa chilena. Para robustecer el conocimiento generado a partir de este estudio exploratorio, es necesario continuar esta línea de investigación acerca de las trayectorias directivas, a fin de informar adecuadamente las decisiones de política y práctica que se tomen en distintos niveles del sistema escolar para asegurar que cada escuela y liceo cuente con un excelente líder. Tal como sucede en la historia de Animales fantásticos y dónde encontrarlos, estos directores fantásticos ya están en nuestras escuelas y liceos: la tarea ahora consiste en identificarlos, desarrollarlos y ubicarlos como directivos. 
158 DIRECTORES FANTÁSTICOS Y DÓNDE ENCONTRARLOS: LECCIONES SOBRE LA PROMOCIÓN INTERNA DE DIRECTIVOS ELEGIDOS POR ALTA DIRECCIÓN PÚBLICA EN CHILE - S. Galdames y Á. González

\section{Referencias}

Agrawal, A., Knoeber, C. R., \& Tsoulouhas, T. (2006). Are outsiders handicapped in CEO successions? Journal of Corporate Finance, 12(3), 619-644. https://doi.org/10.1016/j.jcorpfin.2004.04.005

Arthur, M. B. \& Rousseau, D. M. (1996). The boundaryless career: A new employment principle for a new organizational era. New York: Oxford University Press.

Bower, J. L. (2007). Solve the succession crisis by growing inside-outside leaders. Harvard Business Review, 85(11), 90-96.

Briscoe, J. P. \& Hall, D. T. (2006). The interplay of boundaryless and protean careers: Combinations and implications. Journal of Vocational Behavior, 69(1), 4-18. https://doi.org/10.1016/j.jvb.2005.09.002

Bush, T. (2011). Succession planning in England: New leaders and new forms of leadership. School Leadership and Management, 31(3), 181198. https://doi.org/10.1080/13632434.2010.545383

Bush, T. \& Oduro, G. K. T. (2006). New principals in Africa: Preparation, induction and practice. Journal of Educational Administration, 44(4), 359-375. https://doi.org/10.1108/09578230610676587

Campos, F., Balborán, J., Bustos, C., y González, M. (2014). Formación de directores de excelencia: un mismo objetivo, distintas demandas. Perspectiva Educacional, 53(2), 91-111. https://doi. org/10.4151/07189729-Vol.53-Iss.2-Art.256

Cannella, A. \& Lubatkin, M. (1993). Succession as a sociopolitical process: Internal impediments to outsider selection. Academy of Management Journal, 36(4), 763-793. https://doi.org/10.2307/256758

Carlson, R. O. (1961). Succession and performance among school superintendents. Administrative Science Quarterly, 6(2), 210-227. https://doi.org/10.2307/2390755

Clarke, V. \& Braun, V. (2017). Thematic analysis. Journal of Positive Psychology, 12(3), 297-298. https://doi.org/10.1080/17439760.2016.1262613

Connolly, M., Milton, E., Davies, A. J., \& Barrance, R. (2018). Turning heads: The impact of political reform on the professional role, identity and recruitment of head teachers in Wales. British Educational Research Journal, 44(4), 608-625 https://doi.org/10.1002/berj.3450

Denzin, N. K. \& Lincoln, Y. S. (2000). The discipline and practice of qualitative research. En N. K. Denzin \& Y. S. Lincoln (Eds.), Handbook of qualitative research (pp.1-32). London: Sage Publication. 
Department for Education, UK. (2019). Teacher recruitment and retention Strategy. Recuperado de https://www.gov.uk/government/publications/ teacher-recruitment-and-retention-strategy

Donoso, S., Fernández, J., y Reyes, D. (2019). Directores de escuelas públicas chilenas seleccionados por alta dirección removidos de su cargo antes de finalizar su período de gestión: lecciones para América Latina. Education Policy Analysis Archives, 27(April), 43. https://doi. org/10.14507/epaa.27.3798

Earley, P. \& Weindling, D. (2007). Do school leaders have a shelf life? Career stages and headteacher performance. Educational Management Administration and Leadership, 35(1), 73-88. https://doi. org/10.1177/1741143207071386

Edge, K., Galdames, S., \& Horton, J. (2017). Diversity: New leaders and new leadership. En T. Greany \& P. Earley (Eds.), School leadership and education system reform (pp. 211-221). London: Bloomsbury Publishing.

Fink, D. \& Brayman, C. (2004). Principals' succession and educational change. Journal of Educational Administration, 42(4), 431-449. https:// doi.org/10.1108/09578230410544053

Galdames, S., Montecinos, C., Campos, F., Ahumada, L., \& Leiva V. (2018). Novice principals in Chile mobilizing change for the first time. Educational Management Administration E Leadership, 46(2), 318--338. https://doi.org/10.1177/1741143217707520

Galdames, S. (2019). Trabajo duro, una sed por aprender y un poco de suerte: la trayectoria laboral de los directores de la generación milenio en las escuelas públicas de Chile. Perspectiva Educacional, 58(1), 6991. Recuperado de http://www.perspectivaeducacional.cl/index.php/ peducacional/article/view/821

Galdames, S. \& González, A. (2016). The relationship between leadership preparation and the level of teachers' interest in assuming a principalship in Chile. School Leadership \& Management, 36(4), 435451. https://dx.doi.org/10.1080/13632434.2016.1209178

Gates, S., Baird, M., Master, B., \& Chavez-Herrerias, E. (2019). Principal pipelines: A feasible, affordable, and effective way for districts to improve Schools. Principal pipelines: A feasible, affordable, and effective way for districts to improve schools. Santa Monica: Rand Corporation. https:// doi.org/10.7249/rr2666

Guest, D. \& Rodrigues, R. A. (2014). Beyond the duality between bounded and boundaryless careers: New avenues for careers research. Career 
160 DIRECTORES FANTÁSTICOS Y DÓNDE ENCONTRARLOS: LECCIONES SOBRE LA PROMOCIÓN INTERNA DE DIRECTIVOS ELEGIDOS POR ALTA DIRECCIÓN PÚBLICA EN CHILE - S. Galdames y Á. González

Development International, 19(6), 1-9. https://doi.org/10.1108/CDI09-2014-0123

Hargreaves, A. (2005). Leadership succession. The Educational Forum, 69(2), 163-177. https://doi.org/10.2307/2071933

Hargreaves, A., Moore, S., Fink, D., Brayman, C., \& White, R. (2003). Succeeding leaders? A study of principal succession and sustainability. Recuperado de https://docplayer.net/1316358-Succeeding-leaders-astudy-of-principal-succession-and-sustainability.html

Hartle, F. \& Thomas, K. (2003). Growing tomorrow's school leaders: The challenge. Full report. Recuperado de https://dera.ioe.ac.uk/4929/7/ Growing_Tomorrow's_School_Leaders_Redacted.pdf

Heyman, D., Kloves, S., Rowling, J.K., \& Wigram, L. (Productor) y Yates, D. (Director). (2016). Animales fantásticos y dónde encontrarlos [Película]. Reino Unido y Estados Unidos: Warner Brothers.

Irvine, P. A. \& Brundrett, M. (2017). Negotiating the next step. Educational Management Administration E Leadership, 47(1), 74-90. https://doi. org/10.1177/1741143217720457

James, C., Fitzgerald, S., Fellows, T., Goodall, J., Costas, I., Jones, J., ... Goodall, J. (2018). Primary school headteacher recruitment and selection in England: The processes and the problematic aspects. School Leadership E Management, 39(5), 478-495. https://doi.org/10. 1080/13632434.2018.1525699

Johnson, B. \& Licata, J. (1995). School principal succession and teachers on successor effectiveness. Journal of School Leadership, 5(5), 394-417. https://doi.org/10.1177/105268469500500501

Joseph, S. (2009). Planning to grow your own principal preparation programs: Cultivating excellence in tough economic times. Educational Planning, 18(2), 35-41.

Kamp, A., Lambrecht Lund, H., \& Søndergaard Hvid, H. (2011). Negotiating time, meaning and identity in boundaryless work. Journal of Workplace Learning, 23(4), 229-242. https://doi. org/10.1108/13665621111128655

Karaevli, A. (2007). Performance consequences of new CEO 'Outsiderness': Moderating effects of pre-and post-succession contexts. Strategic Management Journal, 28(7), 681-706.

Legore, J. A. \& Parker, L. (1997). First year principal succession: A study of leadership, role, and change. Journal of School Leadership, 7(4), 369385. https://doi.org/10.1177/105268469700700403 
Leithwood, K., Harris, A., \& Hopkins, D. (2008). Seven strong claims about successful school leadership. School Leadership \& Management, 28(1), 27-42. https://doi.org/10.1080/13632430701800060

Meyer, M. J., MacMillan, R. B., \& Northfield, S. (2009). Principal succession and its impact on teacher morale. International Journal of Leadership in Education, 12(2), 171-185. https://doi. org/10.1080/13603120802449660

Miller, A. (2013). Principal turnover and student achievement. Economics of Education Review, 36, 60-72. https://doi.org/10.1016/j. econedurev.2013.05.004

Miskel, C. \& Cosgrove, D. (1985). Leader succession in school settings. Review of Educational Research, 55(1), 87-105. https://doi. org/10.2307/1170408

Montecinos, C., Ahumada, L., Galdames, S., Campos, F., Leiva, M. V., \& Leiva, M. V. (2015). Targets, threats and (dis)trust: The managerial troika for public school principals in Chile. Education Policy Analysis Archives, 23(87), 1-29. https://doi.org/10.14507/epaa.v23.2083

Moorosi, P. (2010). South African female principals' career paths: Understanding the gender gap in secondary school management. Educational Management Administration E Leadership, 38(5), 547-562. https://doi.org/10.1177/1741143210373741

Moorosi, P. (2012). Mentoring for school leadership in South Africa: Diversity, dissimilarity and disadvantage. Professional Development in Education, 38(3), 487-503. https://doi.org/10.1080/19415257.2011.637430

Oplatka, I. (2012). Towards a conceptualization of the early career stage of principalship: Current research, idiosyncrasies and future directions. International Journal of Leadership in Education, 15(2), 129-151 https:// doi.org/10.1080/13603124.2011.640943

Reeves, C. L. (2010). A difficult negotiation: Fieldwork relations with gatekeepers. Qualitative Research, 10(3), 315-331. https://doi. org/10.1177/1468794109360150

Rivero, R., Hurtado, C., y Morandé, Á. (2018). ¿Cuán preparados llegan los directores escolares?: un análisis sobre su formación y trayectorias laborales previas a ejercer su cargo. Calidad en la Educación, 48, 17-49. https://doi.org/10.31619/caledu.n48.478

Santora, J. C., Caro, M. E., \& Sarros, J. C. (2007). Succession in nonprofit organizations: An insider/outsider perspective. SAM Advanced Management Journal, 72(4), 26. 
162 DIRECTORES FANTÁSTICOS Y DÓNDE ENCONTRARLOS: LECCIONES SOBRE LA PROMOCIÓN INTERNA DE DIRECTIVOS ELEGIDOS POR ALTA DIRECCIÓN PÚBLICA EN CHILE - S. Galdames y Á. González

Schulz, J. (2013). Research methods in educational leadership and management. Recuperado de https://www.tandfonline.com/doi/abs/10.1080/1743 727X.2012.752206

Schwartz, K. B. \& Menon, K. (1985). Executive succession in failing firms. Academy of Management Journal, 28(3), 680-686. https://doi. org/10.2307/256123

Seipert, K. \& Baghurst, T. (2014). Contrasting work values of baby boomers and generation X rural public school principals. Public Administration Quarterly, 38(3), 347.

Stevenson, H. (2006). Moving towards, into and through principalship: Developing a framework for researching the career trajectories of school leaders. Journal of Educational Administration, 44(4), 408-420. https://doi.org/10.1108/09578230610676604

Suutari, V., Tornikoski, C., \& Mäkelä, L. (2012). Career decision making of global careerists. International Journal of Human Resource Management, 23(16), 3455-3478. https://doi.org/10.1080/09585192.2011.639026

Tirozzi, G. (2001). The artistry of leadership: The evolving role of the secondary school principal. Phi Delta Kappan, 82(6), 434-439.

Torres, A. C. (2018). Push, pull, tap and switch: Understanding the career decisions of charter school leaders. Leadership and Policy in Schools, 1-19. https://doi.org/10.1080/15700763.2018.1513155

Tran, H., McCormick, J., \& Nguyen, T. T. (2018). The cost of replacing South Carolina high school principals. Management in Education, 32(3), 109118 https://doi.org/10.1177/0892020617747609

Travis, A. (2016) 'Leadership Succession in a semi-rural Local Authority', Scottish College for Educational Leadership, 1(1), pp. 1-14.

Tulowitzki, P. (2013). Leadership and school improvement in France. Journal of Educational Administration, 51(6), 812-835. https://doi.org/10.1108/ JEA-03-2012-0026

Valenzuela, J. P., Allende, C., y Vanni, X. (2018). Trayectoria de los directores chilenos durante la última década: primeros hallazgos para políticas públicas (Nota Técnica No 8). Valparaíso: Líderes Educativos, Centro de Liderazgo para la Mejora Escolar.

Verbruggen, M. (2012). Psychological mobility and career success in the "New" career climate. Journal of Vocational Behavior, 81(2), 289-297. https://doi.org/10.1016/j.jvb.2011.10.010

White, R. E. \& Cooper, K. (2011). Principals in succession. Vol. 1. Dordrecht: Springer Netherlands. https://doi.org/10.1007/978-94-007-1275-1 
Zepeda, S. J., Lanoue, P. D., Price, N. F., \& Jimenez, A. M. (2014). Principal evaluation-linking individual and building-level progress: Making the connections and embracing the tensions. School Leadership and Management, 34(4), 324-351. https://doi.org/10.1080/13632434.20 14.928681

Recibido: 14/06/2019

Aceptado: 18/10/2019 